\title{
Adequação de protocolos de exportação de um sistema de gestão de experimentos neurocientíficos às especificações de dados sem atrito
}

\author{
Adapting the export protocols of a system of \\ neuroscience experiments management to the \\ frictionless data specifications
}

\section{Cassiano Reinert Novais dos Santos ${ }^{1}$ \\ Carlos Eduardo Ribas ${ }^{2}$ \\ João Alexandre Peschanski ${ }^{3}$}

\section{Resumo}

O Neuroscience Experiments System (NES) é um sistema desenvolvido para gerenciar informações de experimentos de neurociência. O módulo de exportação do NES permite que o pesquisador obtenha os dados e metadados de seus experimentos em formatos interoperáveis, porém a compreensão desses

\footnotetext{
Bacharel em Matemática Aplicada e Computacional, Instituto de Matemática e Estatística da Universidade de São Paulo - IME-USP. São Paulo, SP - Brasil. Centro de Pesquisa, Inovação e Difusão em Neuromatemática (CEPID NeuroMat). ORCID: https://orcid.org/0000-0002-9185-0486 | crns13@gmail.com

Mestre em Ciências, Faculdade de Medicina da Universidade de São Paulo - FM-USP. São Paulo, SP - Brasil. European Bioinformatics Institute - EMBL-EBI. ORCID: https://orcid.org/0000-0002-9572-273X | cribas@ebi.ac.uk

Doutor em Sociologia, Department of Sociology - University of Wisconsin-Madison - UW-Madison. Madison, Wisconsin - Estados Unidos. Centro de Pesquisa, Inovação e Difusão em Neuromatemática (CEPID NeuroMat); Faculdade Cásper Líbero. ORCID: https://orcid.org/0000-0002-2352-1787 | japeschanski@casperlibero.edu.br
}

\section{Como citar:}

Santos, C. R. N. dos, Ribas, C. E., \& Peschanski, J. A. (2020). Adequação de protocolos de exportação de um sistema de gestão de experimentos neurocientíficos às especificações de dados sem atrito. Revista Inovação, Projetos e Tecnologias, 8(1), 83-96. https://doi.org/10.5585/iptec.v8i1.16783 
dados nem sempre é simples. Seguindo os conceitos da metodologia ágil, adotamos a filosofia e as especificações técnicas do Frictionless Data com o intuito de reduzir o atrito frequentemente encontrado ao trabalhar com dados. A adoção do Frictionless Data proporcionou uma melhor compreensão dos dados pelos pesquisadores, além de permitir a criação de scripts e programas para realizar a análise dos dados coletados.

Palavras-chave: Tecnologia da Informação; Gestão; Neurociência; Ciência aberta.

\begin{abstract}
The Neuroscience Experiments System (NES) was developed to manage information originated from neuroscience experiments. Through the NES export module, a researcher is able to download experimental data and metadata in interoperable formats; nevertheless, the understanding of what is downloaded is not always a simple task. In accordance with the agile methodology guidelines, we have worked within the Frictionless Data philosophical and technical framework in order to decrease friction that is commonly associated with understanding data and metadata. Working with Frictionless Data may lead to improving research efficiency; it is also an opportunity to create scripts and softwares to improve data analysis.
\end{abstract}

Keywords: Information Technology; Management; Neuroscience; Open science.

\title{
1 Introdução
}

O Neuroscience Experiments System (NES) é um sistema desenvolvido para gerenciar informações de experimentos de neurociência. O principal objetivo do NES é registrar os dados e metadados de cada etapa presente em um experimento. O sistema foi concebido para permitir que os pesquisadores insiram detalhes suficientes em todos os itens do protocolo experimental, permitindo assim a rastreabilidade dos dados e a reprodução do experimento. Um novo modelo para armazenamento de dados brutos foi proposto por Ruiz-Olazar et al. (2016a) durante a criação desta ferramenta.

O NES é um Software Livre disponível sob a licença Mozilla Public License versão 2.0 $0^{1}$ O código fonte está disponível no repositório $\mathrm{GitHub}^{2}$ e a documentação para instalação e uso do sistema está na plataforma de documentação Read the Docs ${ }^{3}$.

O sistema permite o registro de informações sobre o protocolo experimental, tais como os grupos a serem estudados, os critérios utilizados para a seleção dos participantes e os itens de experimento presentes em cada grupo. No NES, os itens de um grupo são chamados de passos e um passo é um tipo de dado que precisa ser coletado. Cada grupo pode ter um ou mais conjunto de passos. Um conjunto de passos é uma forma de organizar os passos de acordo como eles devem ser executados, sequencialmente e/ou em paralelo (Ruiz-Olazar et al., 2016b). Para facilitar a visualização e a compreensão do protocolo experimental, o sistema gera uma imagem que descreve o fluxo de trabalho. A Figura 1 apresenta um protocolo experimental fictício com coleta de EEG e estímulos ao participante sendo executados de forma paralela.

Experimentos de neurociência podem envolver a coleta de diferentes tipos de dados e o NES está preparado para lidar com boa parte deles. Conforme descrito por Braghetto et al. (2018), entre os possíveis dados que podem ser inseridos no sistema estão: Eletroencefalografia (EEG), Eletromiografia (EMG), Estimulação Magnética Transcraniana (EMT ou TMS, na sigla em inglês), estímulos (auditivos, olfativos, visuais, etc.), dados genéricos (estabilometria, cinemática, comportamentais, etc.), dados de respostas de questionários, dados de instruções e tarefas, dados vindos da integração com o Jogo do Goleiro (Stern et al., 2018) e dados dos participantes. 


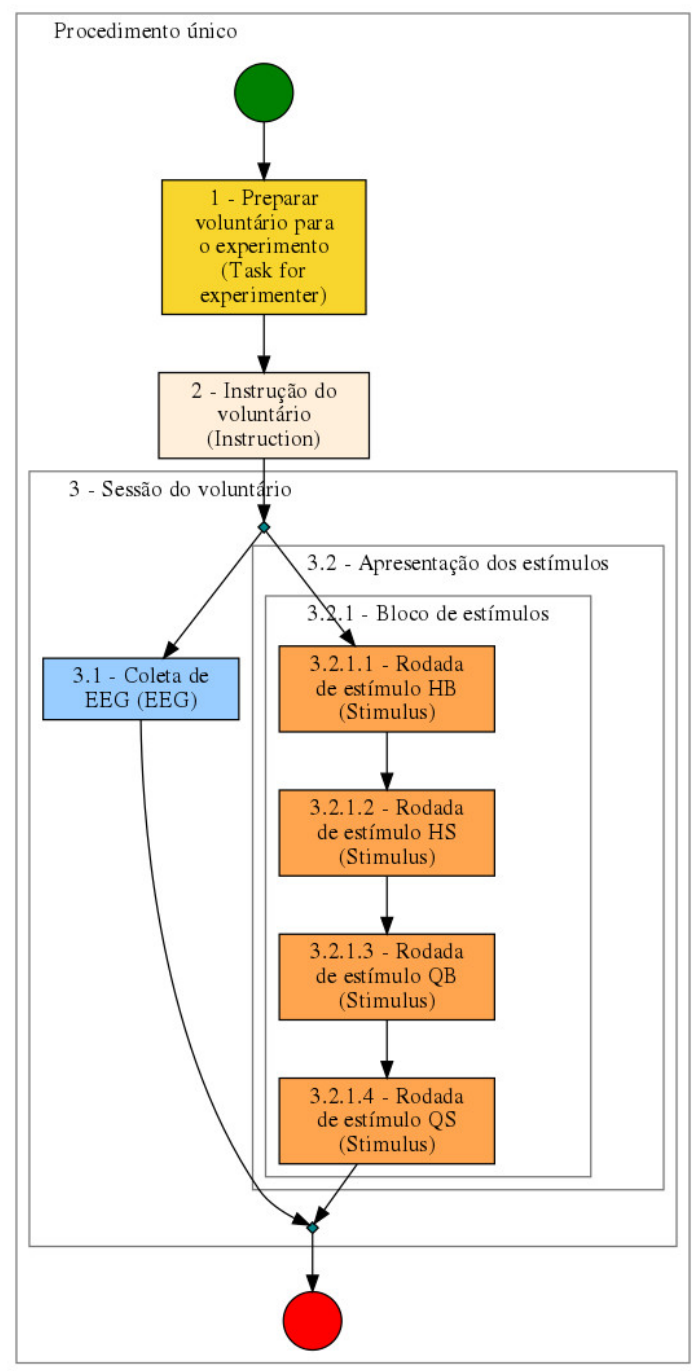

Figura 1 - Imagem gerada pelo NES de um protocolo experimental fictício com coleta de EEG e blocos de estímulos

Fonte: Os autores.

Para o registro de dados de respostas de questionários o NES se integra com o sistema de pesquisas LimeSurvey ${ }^{4}$, um Software Livre para aplicação de questionários online. Sobre os participantes envolvidos no experimento, é possível registrar dados pessoais, dados sociodemográficos, histórico social e avaliações médicas. Os dados podem ser inseridos de forma textual ou como arquivos anexados ao experimento.

O módulo de exportação do NES permite a extração dos dados e metadados em formatos interoperáveis, como CSV, TSV e JSON, que podem ser facilmente utilizados em diferentes plataformas computacionais. Os dados são anonimizados para garantir a segurança dos participantes e para respeitar aspectos éticos e legais. O sistema contempla duas formas de exportação, a primeira é a exportação Por Experimentos, na qual um experimento específico é exportado, a segunda é a exportação Por Participante, na qual apenas dados cadastrais de participantes e questionários respondidos fora de um experimento são exportados.

O objetivo deste estudo é aprimorar o módulo de exportação para atender as especificações de dados sem atrito (frictionless data). Frictionless data é um conjunto de padrões para a publicação de dados, especificamente a aquisição, o compartilhamento e a validação de dados, estabelecendo um padrão que simplifique a abertura de bases de dados (Fowler, Barratt, \& Walsh, 2018). Essas 
especificações foram estabelecidas pela Open Knowledge Foundation, no sentido de apoiar tecnicamente estratégias de ciência aberta.

Entende-se ciência aberta como um conjunto de práticas para a abertura e comunicação pública e livre de processos científicos. É considerado que essas práticas potencializam e aceleram a atividade científica (Santos, 2019).

A filosofia do frictionless data alinha-se diretamente à noção de ciência aberta e de certo modo coloca-a em prática, no que diz respeito ao compartilhamento de dados, um dos elementos centrais da prática científica aberta e livre (Vargas \& Kon, 2014). São elementos autodeclarados da filosofia de dados sem atrito: a simplicidade, a extensibilidade, a cibercultura (edição humana e usabilidade por máquina), o reuso e a interoperabilidade tecnológica. ${ }^{5}$

As especificações de dados sem atrito são adotadas para garantir a reprodutibilidade de experimentos com controle de versões de dados exportados (Yenni et al., 2019) e a padronização de nomenclaturas (Wiese et al., 2019). Outras qualidades potenciais dessas especificações são a estruturação de dados sem erros automáticos gerados por planilhas eletrônicas (LibreOffice Calc, Excel, etc.) e a eficácia na interoperabilidade de bases de dados (Fowler et al., 2017). Vale notar que o modelo frictionless data foi também criticado por não adotar integralmente princípios da web semântica (Sefton, Carragáin, Goble, \& Soiland-Reyes, 2019).

\section{Material e método}

Foi adotado o modelo de desenvolvimento de software conhecido como Metodologia Ágil ${ }^{6}$ para a adaptação do módulo de exportação do NES à filosofia do Frictionless Data. Seguindo os princípios do desenvolvimento ágil, a equipe de desenvolvedores estudou os requisitos e criou uma lista de tarefas. A execução das tarefas era monitorada por meio de reuniões semanais. Nessas reuniões eram discutidas as etapas que foram completadas, os desafios encontrados e as etapas que deveriam ser executadas na próxima iteração. Reuniões virtuais com a equipe da Open Knowledge Foundation foram estabelecidas para apresentar os avanços e garantir a corretude da implementação.

Uma característica importante da prática do desenvolvimento ágil, também aplicada no projeto, foi o desenvolvimento baseado em testes unitários (TDD, na sigla em inglês). A implementação incremental das novas tarefas, sempre com o desenvolvimento de testes na fase anterior, minimizou a criação de bugs no sistema.

Também foi utilizada a biblioteca Python Goodtables ${ }^{7}$, um Software Livre disponibilizado pela Open Knowledge Foundation para verificar a consistência da implementação Frictionless Data. Esta ferramenta acrescentou uma camada adicional na robustez dos testes unitários.

O ponto de entrada para a exportação de dados de experimentos no NES é o menu principal, item Exportar. Ao clicar nesta opção o usuário é levado à primeira tela de exportação, onde ele escolhe entre os dois tipos básicos de exportação, Por Experimentos ou Por Participante.

\subsection{Exportação por experimentos}

A Figura 2 mostra as primeiras opções da exportação Por Experimentos, onde o usuário seleciona o estudo, o experimento, os grupos e o tipo da licença de uso para os dados que serão exportados. 


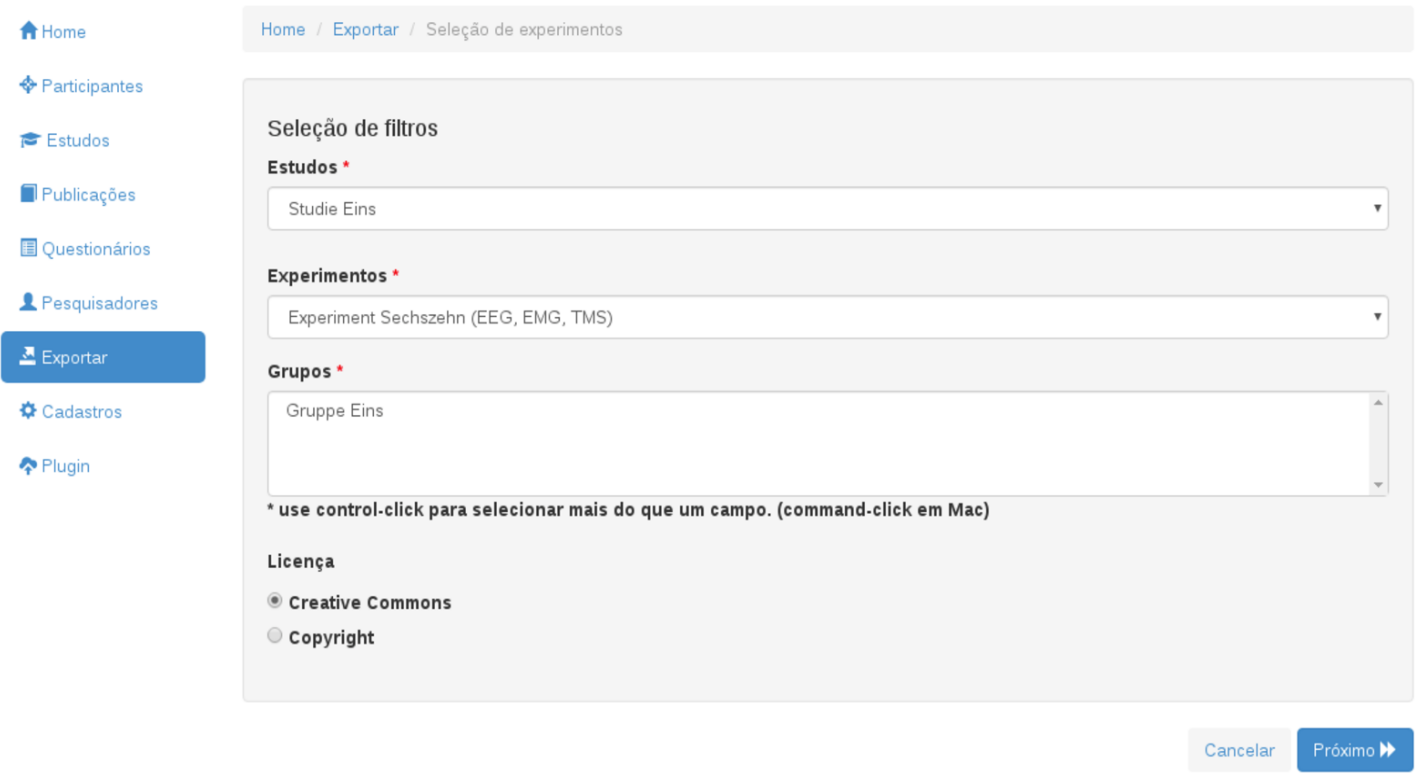

Figura 2 - Tela de exportação de experimentos

Fonte: Os autores.

A próxima etapa da exportação (Figura 3) é composta por 4 abas: Informações Gerais, Questionários respondidos pelos participantes, Questionários dos experimentos e Participantes e diagnósticos.

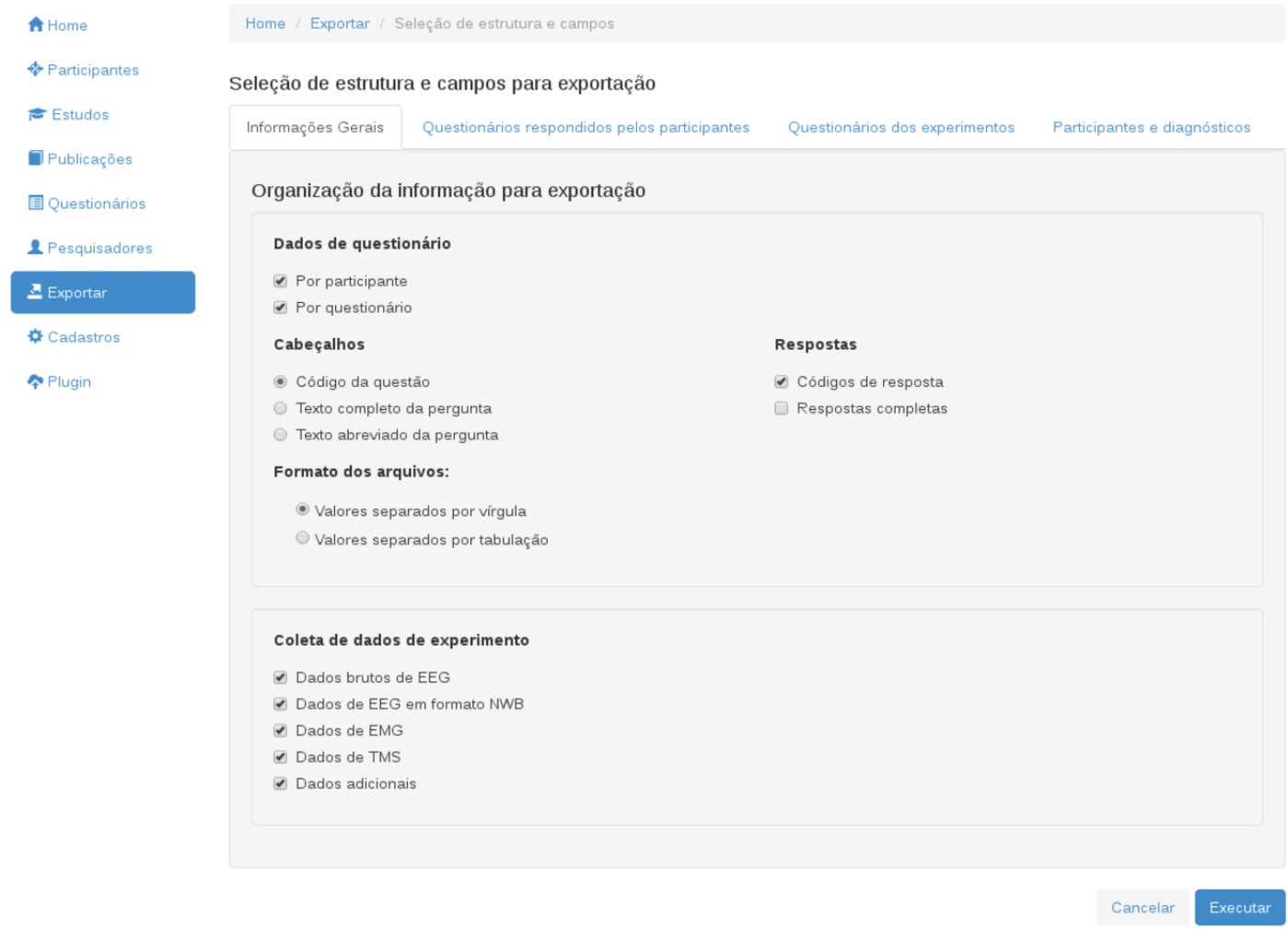

Figura 3 - Tela de exportação de experimentos, aba Informações Gerais

Fonte: Os autores. 
Em Informações Gerais, as seções Dados de questionário, Cabeçalhos, Respostas, Formatos dos arquivos referem-se aos formatos dos dados de exportação de questionários, caso o experimento possua questionários como componentes do Protocolo Experimental. Assim, o usuário pode escolher se as respostas aos questionários serão exportadas agrupadas por participantes individualmente, ou agregadas por questionários; se os cabeçalhos dos arquivos tabulados de respostas conterão os códigos das perguntas, o texto completo das perguntas ou o texto abreviado; se as linhas de respostas virão com os códigos ou com os textos completos (esta opção tem efeito sobre respostas do tipo escolha: múltipla escolha, escolha única entre opções, sim ou não, etc.); e, finalmente, o usuário pode selecionar se os dados tabulados virão no formato csv (comma-separated values, valores separados por vírgula, na sigla em inglês), ou tsv (tab-separated values, valores separados por tabulação, na sigla em inglês).

Na seção Coletas de dados de experimento, o NES mostra os tipos de coletas de dados disponíveis para baixar conforme os componentes constantes do Protocolo Experimental e os arquivos de coletas de dados dos participantes (EEG, TMS, EMG, Coleta Genérica de dados, e Dados Adicionais).

A Figura 4 mostra os questionários disponíveis para exportação provenientes de preenchimentos fora do contexto do experimento. Por exemplo, em casos em que o pesquisador colhe informações dos pacientes antes destes estarem efetivamente participando de um experimento.

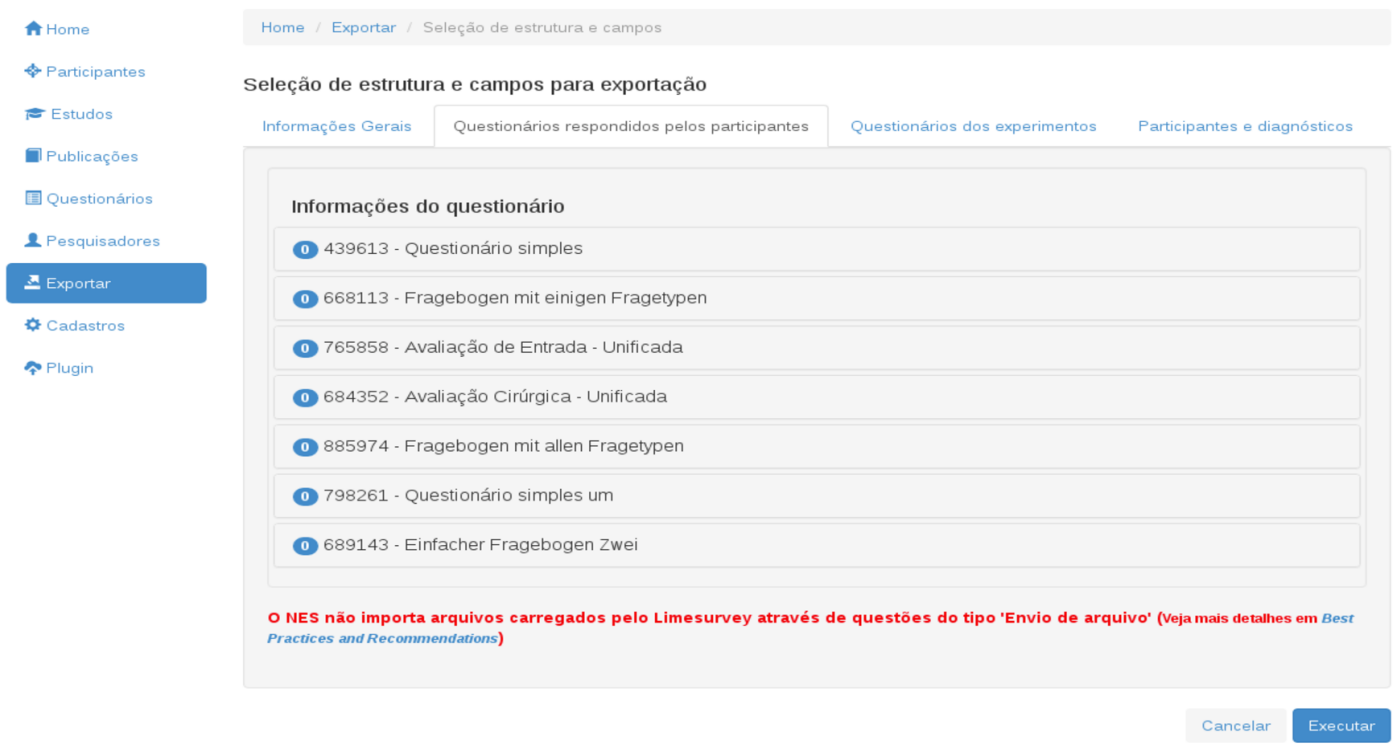

Figura 4 - Tela de exportação de experimentos, aba Questionários respondidos pelos participantes

Fonte: Os autores.

Para cada questionário o usuário pode escolher as questões que ele deseja exportar. A exportação de questionários gera arquivos de texto tabulados contendo as respostas dadas pelos participantes e metadados dos questionários.

O conteúdo da aba Questionários dos experimentos é idêntico ao da aba Questionários respondidos pelos participantes, porém são questionários incluídos como componentes do Protocolo Experimental e não respondidos avulsamente pelos participantes daquele Experimento.

A aba Participantes e diagnósticos (Figura 5) permite ao usuário escolher dentre dezenas de campos relacionados à caracterização dos participantes, incluindo idade, sexo, data de nascimento, 
origem, escolaridade etc. Esses campos correspondem aos dados pessoais, sociodemográficos e história social, que são cadastrados no NES, clicando na opção Participantes no menu principal. Na mesma aba, o usuário pode selecionar campos correspondentes ao diagnóstico dado a cada participante do experimento.

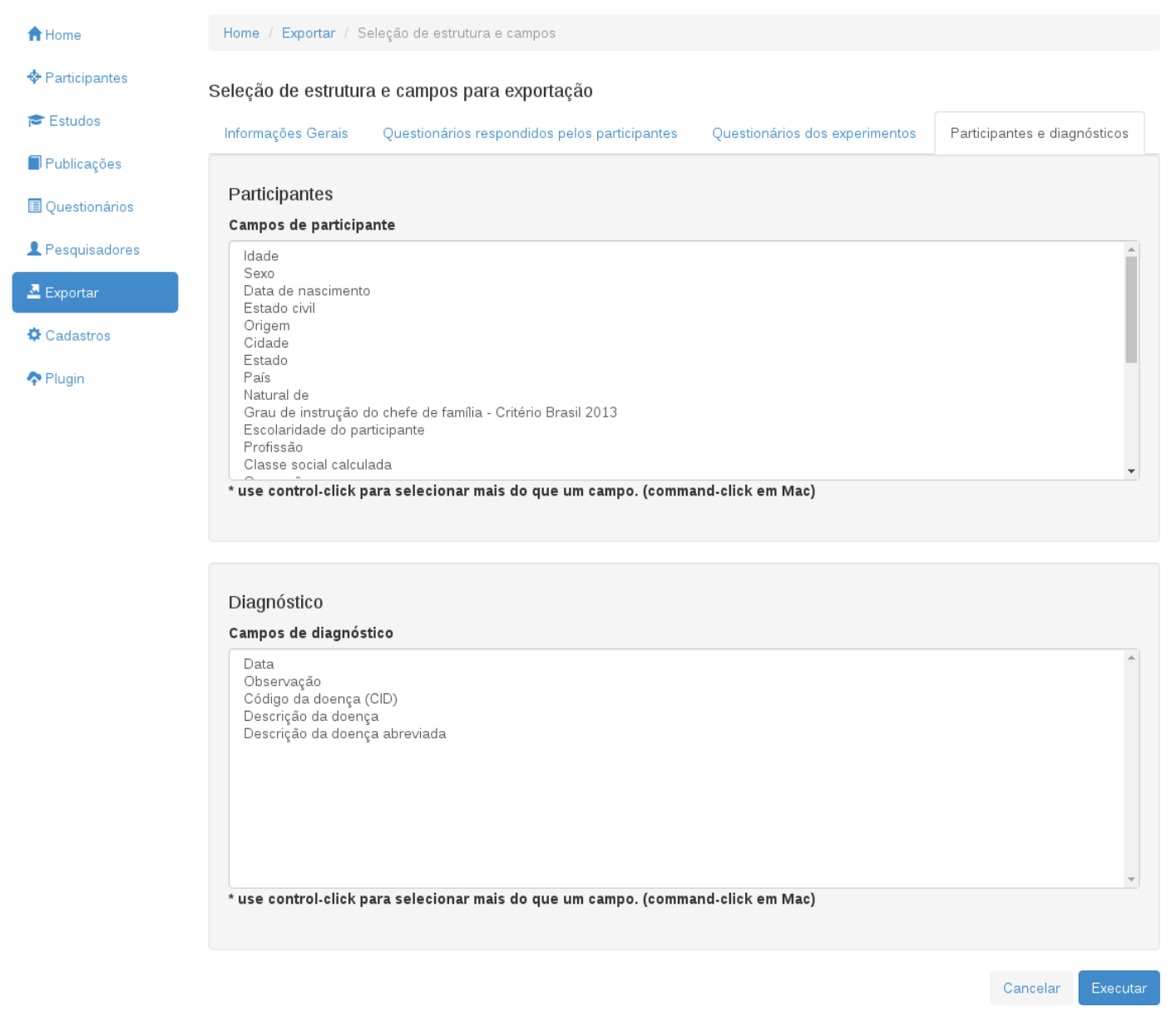

Figura 5 - Tela de exportação de experimentos, aba Participantes e diagnósticos

Fonte: Os autores.

A Figura 6 ilustra a organização dos arquivos e pastas gerados na exportação Por Experimentos antes da implementação do Frictionless Data.

A pasta Experiment_data contém os dados relacionados ao experimento que foi exportado. Ela contém o arquivo Experiment.csv com as informações básicas sobre o experimento. As pastas Group_<SlugTituloGrupo > abrigam todos os dados e metadados do experimento relativos àquele grupo. Abaixo dela está a pasta Experimental_protocol, com as configurações dos aparelhos e/ou materiais usados em passos de EEG, EMG e TMS, configurações da árvore de contexto do Jogo do Goleiro, a descrição textual do protocolo experimental, e uma imagem representativa do fluxo dos passos do protocolo. O passo de Estímulo apresenta uma pasta com o arquivo de estímulo visual. Caso haja arquivos adicionais relacionados aos passos, estes estarão também sob as pastas destes passos, como vemos na pasta Step_1_Instruction. Por fim, a pasta Goalkeeper_game_data contém os arquivos carregados no NES, correspondentes às jogadas executadas pelos participantes no Jogo do Goleiro. 


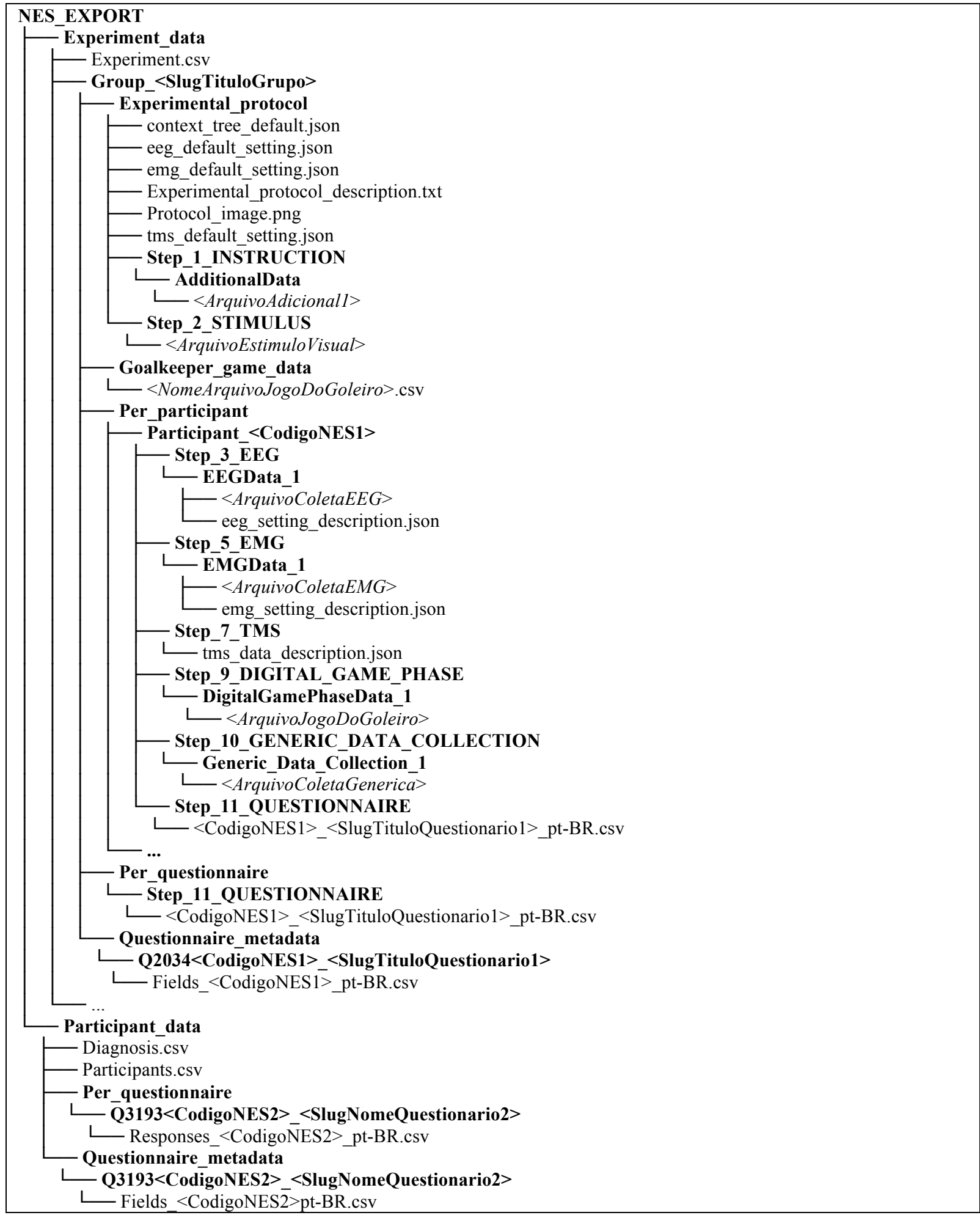

Figura 6 - Árvore de arquivos contidos na exportação para um experimento hipotético com todos os componentes possíveis do Protocolo Experimental*

* Linhas em negrito indicam pasta, linhas sem formatação adicional indicam arquivos nomeados pelo NES, linhas em itálico indicam arquivos nomeados pelo usuário e carregados no NES.

Fonte: Os autores.

Na pasta Per Participant, encontramos os arquivos das coletas de dados referentes aos passos do Protocolo Experimental, para cada participante do experimento. Na pasta Per Questionnaire, estão as respostas dadas aos passos de questionários pelos participantes e os metadados destes questionários. 
A pasta Participant_data contém os arquivos Participants.csv e Diagnosis.csv. O primeiro arquivo lista os atributos dos participantes do experimento, o segundo inclui os diagnósticos de enfermidades atribuídos aos participantes. A pasta Per_questionnaire contém as respostas e a pasta Questionnaire_metadata contém os metadados dos questionários respondidos fora do âmbito do experimento.

\subsection{Exportação por participante}

A exportação Por Participante também produz um arquivo compactado em formato zip, porém ao contrário da exportação Por Experimentos, que contém as informações apenas dos participantes de um determinado experimento, ela contém as informações relativas a todos os participantes cadastrados no sistema (com opção de seleção baseada em alguns critérios) e os questionários respondidos, não vinculados a experimentos.

A exportação de dados de participantes é realizada em duas etapas. A primeira consiste em selecionar os participantes que serão exportados ou exportar todos os participantes do sistema.

A Figura 7 mostra a primeira tela de seleção de participantes, com a opção Participantes selecionados ativada.

Nesta tela o usuário pode escolher entre exportar todos os participantes do sistema ou então selecioná-los com base nos seguintes critérios: Sexo, Estado civil, Localizações, Diagnóstico, e intervalo de Idade.

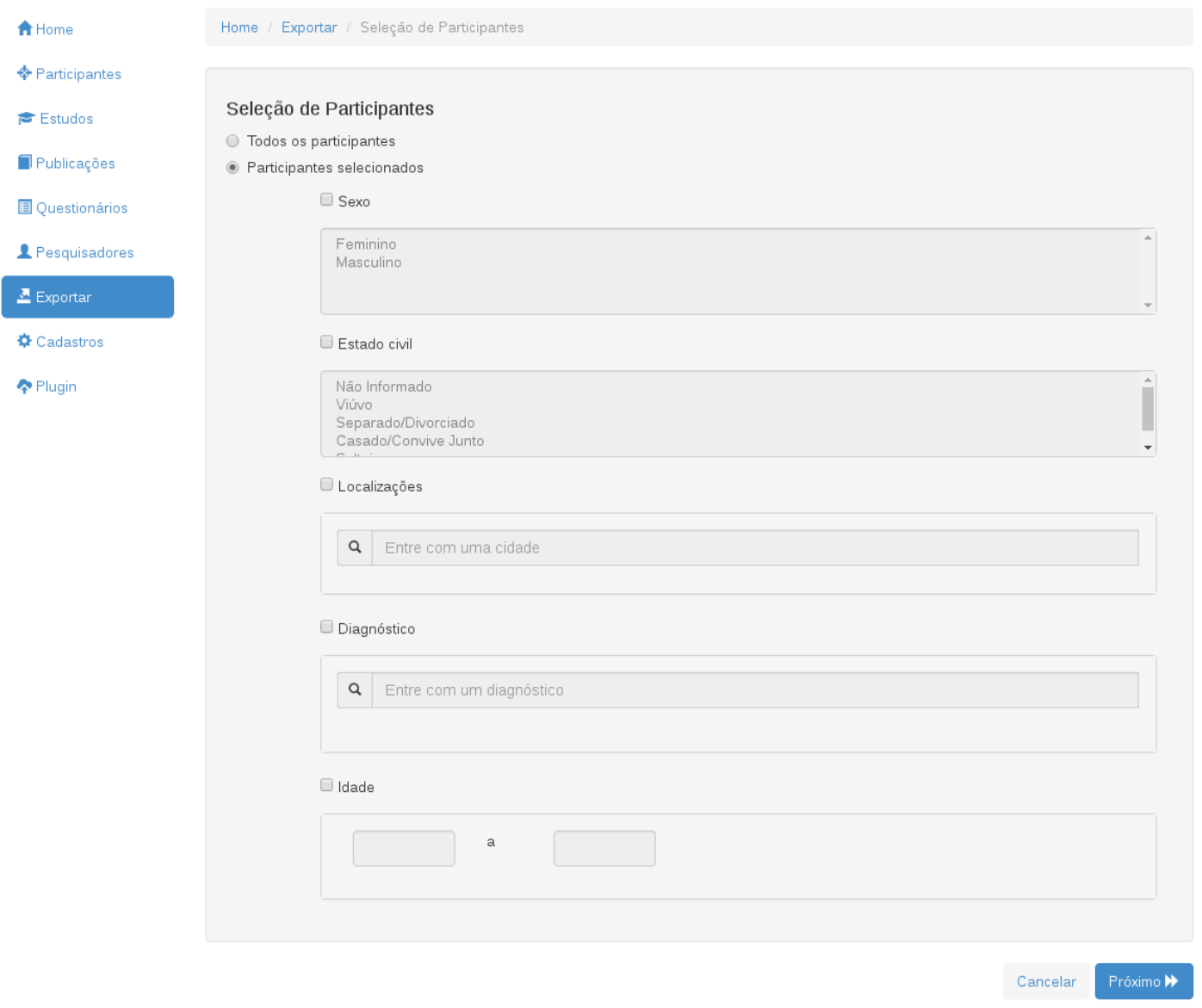

Figura 7 - Tela de exportação de participantes

Fonte: Os autores. 
A Figura 8 mostra a próxima tela da Exportação Por Participante, ela contém opções que definem o formato dos dados dos arquivos correspondentes aos questionários. A seção Informações do questionário mostra uma lista com os questionários que foram respondidos pelos participantes fora dos experimentos. Ao clicar em um dos questionários, a página se expande mostrando os códigos das perguntas que o usuário pode selecionar para exportação.

Nas duas próximas seções, Participantes e Diagnóstico, são listados os campos que representam as informações pessoais dos participantes e diagnósticos.

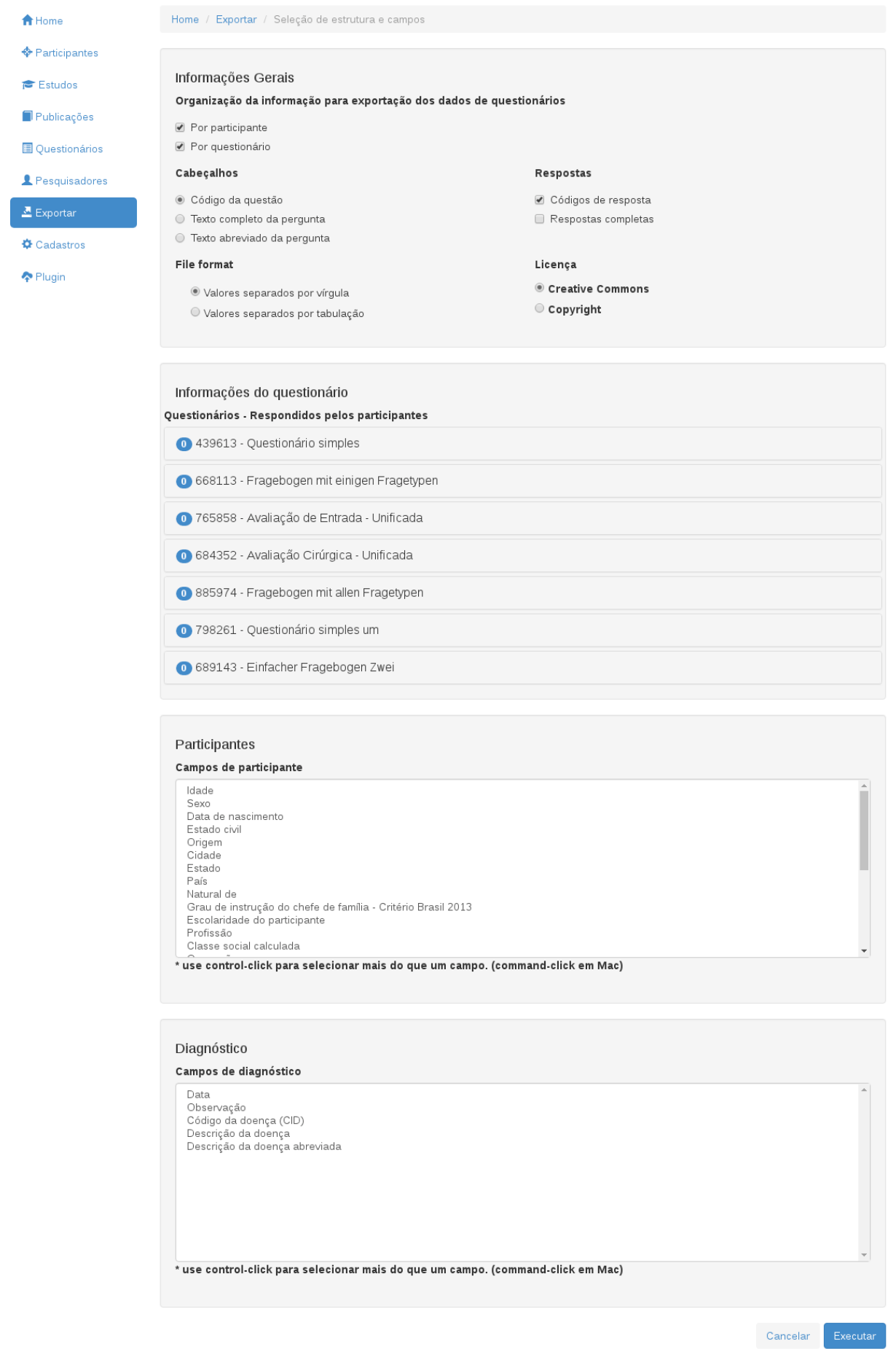

Figura 8 - Tela de exportação de participantes

Fonte: Os autores. 
A Figura 9 mostra uma estrutura de arquivos genérica de uma exportação Por Participante. A exportação define três outras pastas principais: Per_participant, Per_questionnaire e Questionnaire Metadata. A pasta Per_participant possui pastas separadas com as respostas de cada participante. A pasta Perquestionnaire possui um arquivo de respostas para cada questionário, com todas as respostas dos participantes. Por outro lado, a pasta Questionnaire_metadata abriga os arquivos com os metadados de questionários em pastas separadas para cada questionário.

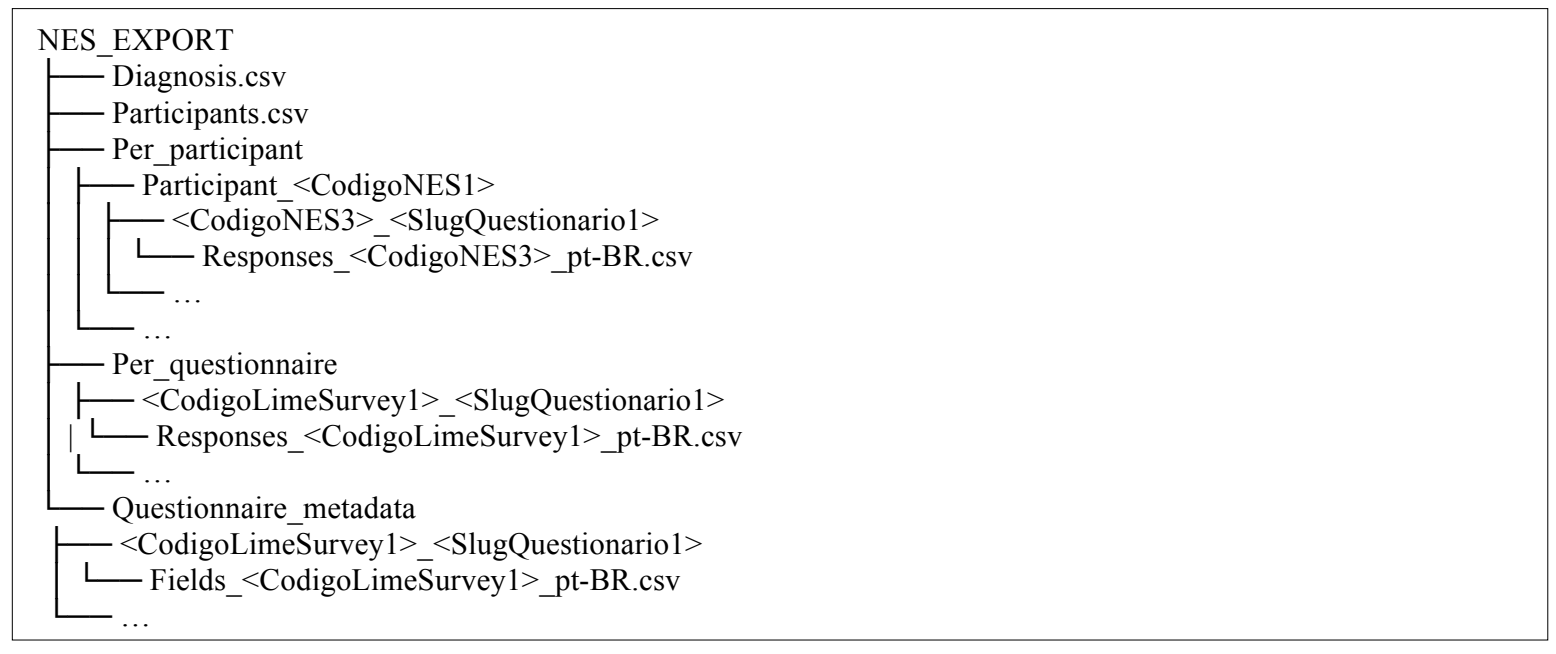

Figura 9 - Árvore de arquivos contidos na exportação Por Participante*

* Linhas em negrito indicam pastas, linhas em texto sem formatação adicional indicam arquivos Fonte: Os autores.

\section{Resultados}

O principal elemento de mudança na exportação é a introdução do arquivo datapackage.json no primeiro nível da árvore de arquivos. Outra mudança é o nome da pasta raiz, que muda de NES_EXPORT para data. A Figura 10 mostra como a árvore de pastas ficou depois das mudanças na construção do arquivo zip.

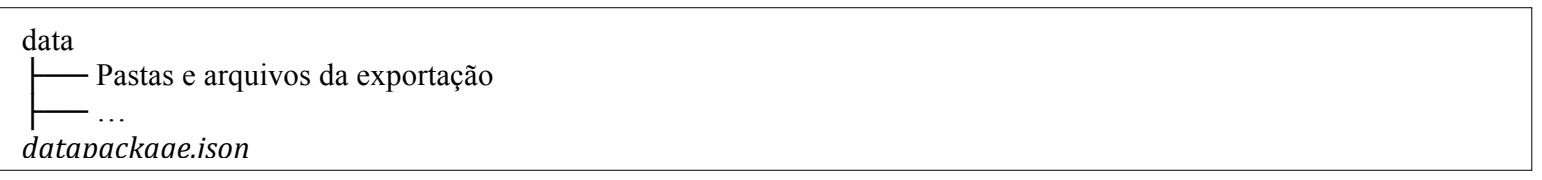

\section{Figura 10 - Alterações na exportação do NES após a aplicação do Frictionless Data,} independentemente do tipo de exportação (Por Experimentos ou Por Participante)

Fonte: Os autores.

Apesar de serem pequenas mudanças, a estrutura do arquivo datapckage.json é a chave para a diminuição da fricção dos dados. Em particular, os dados tabulados são descritos com um alto nível de detalhamento, o que permite a construção de scripts para automatizar a análise de respostas de questionários, dados relativos aos participantes e seus diagnósticos, bem como dos outros tipos de coletas de dados.

O datapackage.json é um descritor com uma propriedade obrigatória e outras opcionais. A propriedade obrigatória, chamada resources, identifica os recursos (pastas/arquivos) contidos na exportação. As outras propriedades que o NES implementa são mostradas na Tabela 1. Estas 
propriedades possuem valores distintos, dependendo do tipo de exportação.

Tabela 1 - Propriedades recomendadas/opcionais pelo Frictionless Data e respectivos valores nas exportações Por Experimentos e Por Participante

\begin{tabular}{|c|c|c|}
\hline Propriedade & Valor Por Experimentos & Valor Por Participante \\
\hline Name & $\begin{array}{l}\text { Título do experimento no NES, com todos os } \\
\text { caracteres minúsculos e sem espaços. } \\
\text { Palavras são separadas por traços }\end{array}$ & $\begin{array}{l}\text { Fixo: "questionnaires-answered-by- } \\
\text { participants-outside-experiment-scope" }\end{array}$ \\
\hline Title & Título do experimento no NES & $\begin{array}{l}\text { Fixo: "Questionnaires Answered by } \\
\text { Participants Outside Experiment Scope" }\end{array}$ \\
\hline Description & Descrição do experimento no NES & $\begin{array}{l}\text { Fixo: 'Export made "Per Participant": the } \\
\text { files contain metadata and responses of } \\
\text { questionnaires filled outside any experiment } \\
\text { in the system. They can be entrance } \\
\text { questionnaires.' }\end{array}$ \\
\hline Contributors & $\begin{array}{l}\text { Os pesquisadores envolvidos no } \\
\text { experimento, além do pesquisador } \\
\text { responsável pelo Estudo }\end{array}$ & $\begin{array}{l}\text { Os responsáveis (criadores) dos } \\
\text { questionários no LimeSurvey. Lista dos } \\
\text { responsáveis, contendo as propriedades title } \\
\text { (nome do questionário no LimeSurvey), } \\
\text { questionnaire (título do questionário no } \\
\text { LimeSurvey), e-mail. }\end{array}$ \\
\hline Created & \multicolumn{2}{|c|}{ Data/hora de criação da exportação } \\
\hline Licenses & \multicolumn{2}{|c|}{$\begin{array}{l}\text { Lista das licenças: na atual versão do NES o usuário pode escolher entre dois tipos de } \\
\text { licenças: Creative Commons ou Copyright. }\end{array}$} \\
\hline Homepage & URL do experimento & - \\
\hline
\end{tabular}

Fonte: Os Autores.

O NES utiliza algumas propriedades opcionais para complementar a descrição dos recursos. A Tabela 2 detalha o uso das propriedades aninhadas na propriedade resources.

Tabela 2 - Propriedades aninhadas na propriedade "resources" e seu uso, de acordo com o tipo de arquivo

\begin{tabular}{ccc}
\hline Propriedade & Descrição & Tipos de arquivos exportados no NES \\
\hline Name & Identificador único do recurso & Todos \\
\hline Profile & Valor: "tabular-data-resource” & Dados tabulados: arquivos csv, tsv \\
\hline Title & $\begin{array}{c}\text { Valor: geralmente o nome do arquivo sem a } \\
\text { extensão. }\end{array}$ & Todos \\
\hline Description & $\begin{array}{c}\text { Identifica o tipo de recurso: usado para } \\
\text { identificar os arquivos de coleta de dados }\end{array}$ & Arquivos relacionados a coletas de dados \\
\hline Format & Extensão do arquivo exportado & Todos \\
\hline Mediatype & Valor: "mediatype/mimetype" & txt, csv, tsv, png, json \\
\hline Encoding & Valor: "UTF-8" & csv, tsv \\
\hline Schema & $\begin{array}{c}\text { Conjunto de propriedades que detalham } \\
\text { características de dados tabulados }\end{array}$ \\
\hline
\end{tabular}

Nota. Os mediatypes são administrados pela Internet Assigned Numbers Authority (IANA).

Fonte: Os autores.

Arquivos com dados tabulados possuem a propriedade schema. Esta propriedade identifica os tipos de dados que estão nas colunas das tabelas, bem como o formato destes tipos de dados. A 
Tabela 3 mostra os campos que são utilizados no NES.

Tabela 3 - Propriedades pertencentes à propriedade schema

\begin{tabular}{|c|c|c|}
\hline Propriedade & Descrição & Valores \\
\hline Type & Tipo do campo & string, number, date, datetime \\
\hline Format & Formato do tipo do campo & $\begin{array}{c}\text { Default para string, number e date; } \% Y \text { - } \\
\% m-\% d \% H: \% M: \% S \text { para datetime }\end{array}$ \\
\hline Name & $\begin{array}{l}\text { Nome do campo (cabeçalho da coluna na } \\
\text { tabela) }\end{array}$ & - \\
\hline Title & $\begin{array}{l}\text { No NES esta propriedade é idêntica à } \\
\text { propriedade name }\end{array}$ & - \\
\hline
\end{tabular}

Nota. A lista completa de valores pode ser vista em https://frictionlessdata.io/specs/table-schema. Acesso em 28 out. 2019. Fonte: Os autores.

\section{Considerações finais}

A adequação do NES às especificações de dados sem atrito foi realizada por meio da inclusão do arquivo datapackage.json aos dois tipos de exportação de dados existentes: a exportação Por Experimentos e a exportação Por Participante. Este arquivo faz o mapeamento de todos os dados e metadados que foram exportados.

$\mathrm{O}$ arquivo datapackage.json permite ao pesquisador e sua equipe desenvolver ferramentas computacionais de análise dos dados que geram resultados automaticamente, conforme a evolução destes experimentos. Estas ferramentas, junto com os dados exportados podem ser compartilhadas com seus pares, automatizando um processo de análise que seria manual quando não se tem uma implementação nos moldes do frictionless data.

A ciência aberta convive com vários desafios, contudo ficou claro que a adoção de práticas reconhecidas pode melhorar a interoperabilidade e o compartilhamento de dados de um sistema. Agora o NES entra no rol das aplicações que implementam as especificações de dados sem atrito, o que o prepara para ser uma alternativa livre e robusta para o gerenciamento e compartilhamento de experimentos em neurociências.

\section{Reconhecimento de apoios}

Esta pesquisa integra o Centro de Pesquisa, Inovação e Difusão em Neuromatemática (CEPID NeuroMat), projeto da Fundação de Amparo à Pesquisa do Estado de São Paulo (FAPESP) 2013/07699-0. O desenvolvimento do NES foi apoiado pelo Frictionless Tool Fund Grant (rec. JAP e CER). A pesquisa de JAP é apoiada pelo Centro Interdisciplinar de Pesquisa da Faculdade Cásper Líbero. Os autores são gratos a Antonio Carlos Roque da Silva Filho e Lilly Winfree pela leitura crítica da versão inicial deste artigo.

\section{Notas}

1 Disponível em: <https://www.mozilla.org/en-US/MPL/2.0/>. Acesso em 31 de maio de 2020.

2 Disponível em: <https://github.com/neuromat/nes>. Acesso em 31 de maio de 2020.

3 Disponível em: <https://nes.readthedocs.io/en/latest/>. Acesso em 31 de maio de 2020.

4 Disponível em: <https://www.limesurvey.org >. Acesso em 31 de maio de 2020.

5 Disponível em: <https://frictionlessdata.io/specs/>. Acesso em 28 de outubro de 2019.

6 Disponível em: <https://en.wikipedia.org/wiki/Agile_software_development>. Acesso em 25 maio 2020.

7 Disponível em: <https://github.com/frictionlessdata/goodtables-py>. Acesso em 25 maio 2020. 


\section{Referências}

Braghetto, K. R., Rocha, E. S., Ribas, C. E., Dos Santos, C. R. N., Rabaça, S. S., \& Ruiz-Olazar, M. (2018, julho). Uma Plataforma Computacional para a Construção de Bancos de Dados para Experimentos de Neurociência. Anais do Brazilian e-Science Workshop (BreSci). Brazilian e-Science Workshop (BreSci), Natal, Rio Grande do Norte. http://natal.uern.br/eventos/csbc2018/?page_id=216

Fowler, D., Barratt, J., \& Walsh, P. (2018). Frictionless Data: Making Research Data Quality Visible. International Journal of Digital Curation, 12(2), 274-285. https://doi.org/10.2218/ijdc.v12i2.577

Ruiz-Olazar, M., Rocha, E. S., Rabaça, S. S., Ribas, C. E., Nascimento, A. S., \& Braghetto, K. R. (2016a). A Review of Guidelines and Models for Representation of Provenance Information from Neuroscience Experiments. In M. Mattoso \& B. Glavic (Orgs.), Provenance and Annotation of Data and Processes (p. 222-225). Springer International Publishing.

Ruiz-Olazar, M., Rocha, E. S., Rabaça, S. S., Ribas, C. E., Vargas, C. D., Nascimento, A. S., \& Braghetto, K. R. (2016b). NES: a free software to manage data from neuroscience experiments. 27-29. https://doi.org/10.3389/conf.fninf.2016.20.00043

Santos, J. C. F. dos. (2019). A ciência aberta e suas (re)configurações: Políticas, infraestruturas e prática científica [Tese (doutorado), Unicamp]. http://repositorio.unicamp.br/jspui/handle/REPOSIP/333948

Sefton, P., Carragáin, E. Ó., Goble, C., \& Soiland-Reyes, S. (2019, outubro 24). Introducing RO-Crate: Research object data packaging. eResearch Australasia Conference, Brisbane, Austrália. https:/conference.eresearch.edu.au/wp-content/uploads/2019/08/2019-eResearch_103_-Introducing-ROCrate-research-object-data-packaging.pdf

Stern, R. B., d’Alencar, M., Uscapi, Y. L., Gubitoso, M. D., Roque, A. C., Helene, A. F., \& Piemonte, M. E. P. (2018). Goalkeeper Game: A New Assessment Tool for Prediction of Gait Performance Under Complex Condition in People With Parkinson's Disease. bioRxiv, 400457. https://doi.org/10.1101/400457

Vargas, C. D. \& Kon, F. (2014). Em defesa do compartilhamento público de dados científícos. Le Monde Diplomatique Brasil, 32, 33.

Wiese, F., Schlecht, I., Bunke, W.-D., Gerbaulet, C., Hirth, L., Jahn, M., Kunz, F., Lorenz, C., Mühlenpfordt, J., Reimann, J., \& Schill, W.-P. (2019). Open Power System Data - Frictionless data for electricity system modelling. Applied Energy, 236, 401-409. https://doi.org/10.1016/j.apenergy.2018.11.097

Yenni, G. M., Christensen, E. M., Bledsoe, E. K., Supp, S. R., Diaz, R. M., White, E. P., \& Ernest, S. K. M. (2019). Developing a modern data workflow for regularly updated data. PLOS Biology, 17(1), e3000125. https://doi.org/10.1371/journal.pbio.3000125 